\title{
PRIME NOTE IN TEMA DI COMUNICAZIONE DI EMERGENZA
}

\author{
di Silvia Illari
}

\section{Inquadramento del tema}

Una disamina di diritto pubblico dedicata alla comunicazione di emergenza pare incontrare difficoltà, stante la scarsità di norme e l'attenzione ancora inziale della teoria. Nei suoi termini generali, si tratta di un tema talora esaminato negli studi circa il cd. crisis management aziendale, disciplina nella quale anzitutto la comunicazione attiene alla gestione di situazioni di crisi, allo scopo di contenere gli effetti negativi sulle attività e la reputazione di un'organizzazione, impresa o industria $^{1}$. Invero, è da aggiungere che, secondo gli insegnamenti della stessa disciplina, si rende necessario che la comunicazione di crisi sia tempestiva ma predisposta in forma pianificata, posto che sono da evitare reazioni disordinate e confuse, tanto da aggravare la crisi anziché favorire la ripresa ${ }^{2}$.

Dipartimento di Scienze Politiche e Sociali, Università di Pavia.

Questo saggio è dedicato al prof. Fulco Lanchester, emerito di Diritto costituzionale italiano e comparato nell'Università di Roma "La Sapienza".

1 In proposito si v. L. LAGADEC, Crisis management: come affrontare e gestire emergenze e imprevisti, Milano, FrancoAngeli, 2002; L. NorSA, Risk, Issues e Crisis management, Milano, Ipsoa, 2009; E. InvernizzI, R.C. GAmBetTI, La comunicazione per la gestione delle crisi, in E. INVERNIZZI, S. ROMENTI (a cura), "Relazioni pubbliche e corporate communication", Milano, McGraw-Hill, 2012, p. 139; L. Poma, G. Vecchiato, La guida del Sole 24 Ore al crisis management. Come comunicare la crisi: strategie e case history per salvaguardare la business continuity e la reputazione, Milano, Il Sole 24 Ore, 2012.

2 Anche di recente P. PiciocchI, Crisis Management e Crisis Communication. La rilevanza della comunicazione nella gestione delle crisi d'impresa, Torino, Giappichelli, 2018, p. 33. 
Inoltre, il tema ha costituito oggetto di trattazioni di sociologia, specialmente del ramo designato come sociologia della comunicazione 3 . In particolare, si segnala l'interesse più generale degli studi sociologici per la crescente percezione del rischio e dell' incertezza nella vita civile contemporanea. Comunemente pare intervenuta la consapevolezza che le emergenze non siano condizioni eccezionali ma rispondano a un fenomeno ricorrente nell'attuale tipo di società, alimentando richieste di sicurezza da parte dei consociati ${ }^{4}$. In limine si aggiunga che, nel contempo, è anche diffusa una certa resistenza psicologica all'accettazione del rischio, a causa di aspettative nella scienza e nella tecnica che inducono l'uomo di oggi a ritenere che ogni evento possa essere previsto nella sua individualità e quindi che possano riconoscersi anche i fattori responsabili dei singoli eventis.

Può anche risultare utile qualche riferimento di diritto comparato. Bisogna pur notare che nel dibattito scientifico internazionale la crisis communication, oltre i limiti degli studi aziendali, ha assunto un tratto

3 Nella letteratura sociologica v. p. es. G. MAzzolEni, Comunicare nell'emergenza: un problema in più per lo Stato, in G. MAZzolEnI, "Comunicazione e potere", Napoli, Liguori, 1992; p. 120. R. SAVArese (a cura), Comunicazione e crisi, Milano, FrancoAngeli, 2002; M. Lombardi, Comunicare nell'emergenza, Milano, Vita e Pensiero, 2005; A. CeRASE, Rischio e comunicazione. Teorie, modelli e problemi, Milano, Egea, 2017 ma anche A. CERASE, Quale idea della comunicazione del rischio? Tra teoria, prassi e assunti impliciti, in A. Amato, A. Cerase, F. Galadini, "Terremoti, comunicazione, diritto. Riflessioni sul processo alla "Commissione Grandi Rischi" ”, Milano, FrancoAngeli, 2015, p. 145.

4 Si ricorda la teorizzazione della cd. Risikogesellschaft da parte del sociologo tedesco Ulrich Beck, per il quale l'attuale dimensione sociale del rischio è il portato del processo di modernizzazione, specie per alcuni fattori come la globalizzazione, la trasformazione del lavoro, lo sviluppo tecnologico e scientifico. Si v. U. BECK, La società del rischio. Verso una seconda modernità, Roma, Carocci, 2002, p. 31 ma anche Z. BAUMAN, La società dell 'incertezza, Bologna, il Mulino, 1988; N. LuHMann, Sociologia del rischio, Milano, Mondadori, 1996.

5 Sul punto F. Politi, La sentenza del Tribunale dell'Aquila sulla responsabilità dei membri della Commissione "Grandi Rischi". Posizione del tema, testo della relazione presentata al XII Convegno Nazionale di Diritto Sanitario "Eternit - Stamina - L'Aquila: tre casi difficili tra scienza e diritto", Alessandria 14 Novembre 2014. In tema di scienza e tecnica quali fattori idonei a riformare le visioni generali della vita e dell'uomo si rimanda a P.G. Grasso, Costituzione, scienza, tecnica. Brevi note, in S. Illari (a cura), "La Costituzione italiana sessant'anni dopo. Esperienze e prospettive", numero monografico della rivista Il Politico, n. 3, 2009, p. 75. Va ricordata l'osservazione secondo cui la scienza appare trasformarsi da attività puramente speculativa a mera "registrazione dei fatti", da cui trarre previsioni utili. Anche la tecnica, riferita alla fase pratica, pare idonea a fare tutto (P.G. GRASso, Costituzione, scienza, tecnica. Brevi note, cit. p. 76). 
multidisciplinare. In particolare, negli Stati Uniti si riscontrano indirizzi di studio consolidati anche per l'azione di governo e della pubblica amministrazione, specialmente circa la comunicazione del rischio per calamità naturali o di origine antropica ${ }^{6}$.

Negli Stati Uniti poi la materia ha trovato propria regolamentazione, nell'ambito della cd. risk regulation, da parte di alcune Agenzie dell'Esecutivo federale, che da tempo, su impulso del Congresso e in collaborazione con la comunità scientifica, hanno introdotto regole e procedure di analisi del rischio nel settore della tutela ambientale e della salute ${ }^{7}$. Si può quindi menzionare una diffusa attività di pianificazione, determinata anche secondo apposite linee-guida di tipo operativo8 ${ }^{8}$. Nell'ordinamento dell'Unione europea la risk regulation ha avuto ulteriori sviluppi e al presente l'analisi del rischio costituisce principio generale della legislazione sulla sicurezza alimentare: ivi è ricompresa anche la comunicazione delle attività

6 Per una disamina della dottrina statunitense si v. NATIONAL RESEARCH COUNCIL, Improving Risk Communication, Washington DC, National Academy Press, 1989; S.G. HADDEN, Institutional Barriers to Risk Communication, in 9 Risk Analysis 301 (1989); P.M. Sandman, Crisis Communication Best Practices: Some Quibbles and Additions, in 34 (3) J. Appl. Comm. Research 257 (2006); T. CoomB, Information and Compassion in Crisis Responses: A Test of their Effects, in 8 J. Publ. Relations Research 5 (1999); ); T. CoomB., Ongoing Crisis Communication: Planning, Managing and Responding, Los Angeles CA, Sage, 2007; B. Reynolds, J. Galdo, L. SoKler, Crisis and Emergency Risk Communication, Atlanta GA, Center for Disease Control and Prevention, 2002; P. ARGENTI, Crisis Communication: Lesson from 9/11, in 80 Harv. Bus. Rev. 103 (2002); B. REYNOLDS, M. W. SEEGER, Crisis and Emergency Risk Communication as an Integrative Model, in 10 J. Health Comm. 43 (2005); M.J. Palenchar, Historical Trends of Risk and Crisis Communication, in R.L. Heath, H. Dan O'HaIR (eds.), "Handbook of Risk and Crisis Communication", New York NY, Routledge, 2009, p. 31, con riferimento specifico alle azioni intraprese dal Governo federale.

7 V.T. Covello, F.H. Hallen (eds.), Seven Cardinals Rules of Risk Communication, Washington DC, U.S. Environmental Protection Agency, 1988; più recente U.S. ENVIRONmental Protection Agency, Risk Communication, U.S. EPA, Washington, Office of Policy Analysis, 2013. Per approfondimenti si rimanda a S. Illari, La tutela ambientale ripartita, Padova, Cedam, 2002, p. 193.

8 Circa la pianificazione della comunicazione di emergenza negli Stati Uniti v. V.T. Covello, D.B. McCallum, M.T. Pavlova (eds.), Effective Risk Communication. The Role and Responsibility of Government and Nongovernment Organization, New York, Plenum Press, 1989 e in particolare i seguenti saggi ivi contenuti: M. BARAM, Risk Communication: Moving from Theory to Law to Practice, p. 37; P.D. GALBRAITH, Interactions between State and Federal Programs, p. 73; T. WILSON, Interactions between Community/ Local Government Programs, p. 77. 
verso l'esterno'. Si aggiunga che nelle norme di diritto comunitario la comunicazione del rischio rivolta alla popolazione risulta per la prima volta regolata con la cd. direttiva "Seveso I" del 1982, in materia di prevenzione degli incidenti industriali rilevanti ${ }^{10}$.

Quanto al contesto internazionale, è da rilevare che dal 2005 l'Organizzazione mondiale della sanità (OMS) ha prescritto la comunicazione del rischio tra le misure di vitale importanza da assumere durante la gestione di pandemie, crisi umanitarie e disastri naturali, elaborando anche apposite linee-guida ${ }^{11}$. Tanto è previsto poiché informazioni accurate, fornite tempestivamente, consentono alle persone coinvolte e interessate da questi eventi di seguire comportamenti per proteggere se stesse, le loro famiglie e le comunità dai pericoli per la salute e per la vita. In modo corrispondente, dal febbraio 2020 sono stati fissati criteri di natura tecnico-operativa (più volte aggiornati), dovuti alle esigenze insorte per la pandemia da Covid-19, tra l'altro con rinnovato richiamo al principio generale secondo che la comunicazione del rischio è generalmente disposta come strumento per la salute pubblica ed è parte integrante di qualsiasi risposta di emergenza. Ne deriva per gli Stati aderenti la regola di comunicare "rapidamente", "regolarmente" e "in modo trasparente" con la popolazione, costruendo fiducia ${ }^{12}$.

9 Reg. (CE) n. 178/2002, specie l'art. 6, laddove la comunicazione del rischio, definita "scambio interattivo di informazioni e pareri riguardanti gli elementi di pericolo e i rischi" (art. 3), coinvolge anche i consumatori, al fine di tutelare la salute, in un clima di fiducia per il corretto funzionamento del mercato. Per vero, importa notare che nuove regole per garantire una comunicazione del rischio "trasparente, ininterrotta e inclusiva" sono previste dal Regolamento (UE) n. 1381/2019 del 20 giugno 2019 in vigore dal 27 marzo 2021, anche allo scopo di "contribuire a un dialogo aperto e partecipativo" con i consumatori.

${ }^{10}$ Dir. n. 82/501/CEE del 5 agosto 1982.

${ }^{11}$ Stando alle International Health Regulations, fissate dall'oms per la prevenzione, la tutela, il controllo e la risposta di salute pubblica in caso di diffusione transfrontaliera di malattie (art. 2), gli Stati aderenti si impegnano a segnalare l'emergere di eventuali focolai, a condividere le informazioni disponibili e a seguire le indicazioni della stessa Agenzia dell'ONU. In questo contesto generale, si osservi che la seconda parte del regolamento è appositamente titolata "Information and Public Health Response" (artt. 5-14). Si veda WHO, International Health Regulations ( $3^{\mathrm{d}} \mathrm{ed}$.), Geneva, wHO Press, 2016 e pure wHO, Communicating Risk in Public Health Emergencies: A WHO Guideline for Emergency Risk Communication Policy and Practice, 2017. Entrambe le pubblicazioni sono reperibili sul sito web dell'oms.

12 "One of the most important and effective interventions in a public health response to any event is to proactively communicate what is known, what is unknown, and what is 
Ancora l'importanza della comunicazione pubblica nella gestione dell'emergenza risulta confermata per la drammatica esperienza di eventi come terremoti, alluvioni, uragani, frane, pandemie, incidenti industriali, attentati terroristici. In tal senso, in Italia paiono emblematiche le vicende giudiziarie conseguenti ai fatti del terremoto del1'Aquila13. Così risulta anche per la valanga di Rigopiano, il cui processo è ancora in corso. Più in generale, è da notare che in occasione di procedimenti giudiziari, già definiti o a tutt'oggi pendenti, conseguenti a calamità naturali, sono sorte questioni legate anche alla comunicazione: la condotta oggetto del contendere consiste nella (mancata o carente) comunicazione del rischio o del pericolo in atto da parte

being done to get more information, with the objectives of saving lives and minimizing adverse consequences", in WHO, Risk Communication and Community Engagement Readiness and Response to Coronavirus Disease (COVID-19), Interim guidance, 19 marzo 2020, p. 1 , reperibile sul sito web dell'oms.

${ }^{13} \mathrm{Si}$ fa menzione al procedimento iniziato con sentenza Trib. L'Aquila, 22 ottobre 2012 n. 380; poi App. L'Aquila, 10 novembre 2014, n. 3317 e Cass. pen. sez. IV, 25 marzo 2016 n. 12748/16. Furono allora condannati in primo grado e poi assolti i sei membri "tecnici" della Commissione nazionale per la previsione e la prevenzione dei grandi rischi, organo di consulenza del Servizio nazionale della protezione civile. Gli stessi si erano riuniti a L'Aquila pochi giorni prima del terremoto del 6 aprile 2009. Fu invece condannato l'allora vicecapo del Dipartimento della protezione civile, autore di una discussa intervista televisiva ai margini della suddetta riunione, i cui contenuti venivano ritenuti, anche in secondo grado, impropriamente rassicuranti. In estrema sintesi, agli imputati era stato contestato il reato di omicidio colposo plurimo perché, attraverso una carente analisi del rischio nonché una scorretta attività di informazione, avrebbero indebitamente rassicurato la popolazione aquilana, così inducendo una parte di essa a dismettere i consueti comportamenti di risposta al sisma e a restare in casa dove avrebbero trovato la morte. Il testo delle sentenze è pubblicato in Dir. pen. cont., reperibile all'indirizzo internet http://penalecontemporaneo.it. La vicenda giudiziaria ha avuto una forte eco anche nel dibattito internazionale. Vasta è letteratura penalistica. Ex plubribus v. G. ForNASARI, G. InSOLERA, Scienza, rischi naturali, comunicazione del rischio e responsabilità penale. Il punto di vista del penalista, in A. Amato, A. Cerase, F. Galadini, "Terremoti, comunicazione, diritto. Riflessioni sul processo alla Commissione Grandi Rischi”, Milano, FrancoAngeli, 2015, p. 7; A. GALLUCCIO, Comunicazione (scientifica) e responsabilità penale: riflessioni sulla causalità psichica a margine della sentenza "Grandi Rischi", in A. Amato, A. CERASE, F. GALAdinI, "Terremoti, comunicazione, diritto. Riflessioni sul processo alla Commissione Grandi Rischi”, Milano, FrancoAngeli, 2015, p. 293; C. VALBONESI, La colpa trema. Il terremoto dell'Aquila e la condanna agli scienziati della commissione grandi rischi, in Giust. pen., n. 6, 2013, pt. II, 403; C. VALBONESI., Terremoti colposi e terremoto della colpa. Riflessioni a margine della sentenza "Grandi Rischi", in Riv. it. dir. proc. pen., n. 3, 2016, p. 1498; A. MARChInI, La responsabilità penale della Commissione Grandi Rischi per gli eventi lesivi causati dal terremoto dell'Aquila, in Cass. pen., n. 12, 2016, p. 4438. 
delle autorità di protezione civile e degli operatori impegnati nello svolgimento di attività di previsione, prevenzione e gestione delle emergenze, con l'accertamento di eventuali responsabilità penali ${ }^{14}$.

Per le osservazioni che precedono pare da discernere un nesso tra la comunicazione dell'emergenza e la protezione dei diritti fondamentali della persona, il diritto alla salute, il diritto all'integrità e alla vita in primis, riconosciuti e garantiti dalla Costituzione (oltre che dalla Carta dei diritti fondamentali dell'Unione europea e dalla Convenzione europea per la salvaguardia dei diritti dell'uomo). La funzione di informare e di comunicare risponde soprattutto allo scopo di garantire la sicurezza delle popolazioni in pericolo e anche dell' ambiente e dei beni. In termini diversi è pure rilevante la cd. reputation delle pubbliche amministrazioni coinvolte in eventuali situazioni di emergenza.

\section{Circa una nozione empirica di comunicazione di emergenza}

È da tenere presente che nella dottrina di diritto pubblico a tempi remoti risalgono le trattazioni circa i poteri esercitati dalle autorità di governo e di amministrazione in presenza di condizioni storico-ambientali straordinarie, nelle quali alla cura dei pubblici interessi riescano insufficienti i poteri destinati alle condizioni di normalità, secondo le regole stabilite per i tempi ordinari. Per quanto concerne le espressioni che indicano dette condizioni straordinarie o i poteri per esse esercitate, nella teoria risulta applicata la formula "necessità". Più di recente appare adoperato anche il termine "emergenza" 15.

14 V. per es.: Cass. pen. sez. IV, 10 luglio 2001 n. 33577, sui fatti dell'alluvione in Piemonte del 1994, in Riv. pen., 2001, 995. Cass. pen, sez. IV, 3 maggio 2010, n. 16761, sul caso della frana di Sarno del 1998, in Cass. pen., 2011, 82. Trib. Tempio Pausania, 29 settembre 2017, inedita, circa l'alluvione di Olbia del 2013. Cass. pen. sez. IV, 12 aprile 2019 n. 22214, inedita, sull'alluvione del novembre 2011 a Genova. Per considerazioni in proposito si rimanda ai saggi di Amato e Valbonesi contenuti in A. AmATo, G. FlORA, C. VALBONESI (a cura), Scienza, diritto e processo penale nell'era del rischio, Torino, Giappichelli, 2019.

${ }_{15}$ Nella vastissima letteratura innanzitutto: S. Romano, Sui decreti legge e lo stato di assedio in occasione del terremoti di Messina e Reggio Calabria (1909), in Riv. dir. pubbl. e pubbl. amm. in Italia, 1909, p. 251, la cui copia elettronica è reperibile sul "Portale storico della Camera dei Deputati" all'indirizzo Internet www.storia.camera.it (voce "Bibliografia del Parlamento"); G. Miele, Le situazioni di necessità dello Stato, in Arch. dir. pubbl., 1936, p. 377; G. Мотzo, Il comando delle forze armate e la condotta dei regimi di 
In ordine a tali fini, carattere proprio hanno assunto complesse attività di informazione e di comunicazione, compiute dalle pubbliche autorità principalmente attraverso comunicati, bollettini, conferenze stampa, pagine web e messaggi sui social media. In tempi più remoti erano in uso bandi, manifesti, volantini, comunicati radio e annunci per megafono (questi ultimi ripresi da alcuni Sindaci per le fasi più critiche dell'epidemia da Covid-19).

Spesso si tratta di diffondere informazioni alla popolazione su certi eventi e su certi effetti, con l'indicazione di comportamenti e misure di autoprotezione da adottare a tutela della sicurezza individuale e collettiva. Talvolta si trasmettono anche restrizioni all'esercizio di diritti di singoli o di gruppi. Vi sono però anche altri casi in cui si prescrivono a determinate categorie di persone dei comportamenti di collaborazione con le stesse autorità, in ragione di mezzi ovvero di particolari attività svolte di solito da privati. All'esercizio di queste attività sono di frequente correlati obblighi di informazione erga omnes. Oltre alla comunicazione verso l'esterno, particolare rilevo assume anche la comunicazione interna all'amministrazione pubblica, allo scopo di predisporre e far funzionare l'organizzazione dell'emergenza.

È da considerare che negli ordinamenti giuridici la comunicazione pubblica acquista tratti peculiari per la necessità di dare notizia immediata e rapida di atti normativi e di provvedimenti, in modo di conseguire conoscenza effettiva tra $\mathrm{i}$ destinatari ${ }^{16}$. Tale necessità è massimamente

emergenza nel sistema costituzionale italiano, Milano, Giuffrè, 1957; P.G. GRASso, I problemi giuridici dello "stato di assedio" nell'ordinamento italiano, Pavia, Pubblicazioni dell'Università di Pavia, 1959; P.G. Grasso, Necessità, in Enc. dir., XXVII, Milano, 1997, p. 867; V. Angiolini, Necessità ed emergenza nel diritto pubblico, Padova, Cedam, 1986; F. Modugno, D. Nocilla, Problemi vecchi e nuovi sugli Stati di emergenza nell'ordinamento italiano, in Scritti in onore di Massimo Severo Giannini, III, Milano, Giuffrè, 1988, p. 513; P. PINNA, L'emergenza nell'ordinamento costituzionale italiano, Milano, Giuffrè, 1988; A. Pizzorusso, Emergenza (stato di) in Enc. Sc. Soc., III, Roma, 1993, p. 551. Più di recente: G. Marazzita, L'emergenza costituzionale. Definizione e modelli, Milano, Giuffrè, 2003; P. BonetTI, Terrorismo, emergenza e costituzioni democratiche, Bologna, il Mulino, 2006; G. RAzZANo, L'amministrazione dell'emergenza, Bari, Cacucci, 2010; A. CARdone, La "normalizzazione" dell'emergenza. Contributo allo studio del potere extra ordinem del Governo, Torino, Giappichelli, 2011; G. DE MinICO, Costituzione. Emergenza terrorismo, Napoli, Jovene, 2016.

16 Sulla determinazione del rapporto tra conoscenza e principio di pubblicità v. diffusamente P. MARsocci, Poteri e pubblicità. Per una teoria giuridica della comunicazione istituzionale, Padova, Cedam, 2002. 
avvertita nella gestione di situazioni straordinarie, laddove la prima esigenza delle amministrazioni di emergenza è quella di diffondere informazioni e di farle circolare attraverso uno scambio comunicativo con i consociati: senza tale compito qualsiasi azione di intervento e di tutela resterebbe inefficace. La comunicazione dell'emergenza pare dunque essere di tipo particolare, ordinata a realizzare l'interesse generale della collettività, quasi comunicazione pubblica per eccellenza in ragione dei diritti fondamentali che concorre a tutelare.

Si deve anche rilevare che storicamente una siffatta comunicazione si esercita a seguito di eventi eccezionali, con la trasmissione unilateratale di informazioni, comandi, divieti e procedure sia nei confronti dei soggetti dell'apparato amministrativo, impegnati nei soccorsi, sia nei confronti delle popolazioni coinvolte, stante la necessità di conoscenza del comando da parte dei destinatari per l'osservanza.

A questo proposito può riuscire utile il confronto con le regole sulla legge secondo le quali la conoscenza è presupposta come effetto inerente al procedimento di formazione, promulgazione e pubblicazione. È offerta una possibilità diretta di prenderne conoscenza ai cittadini e agli altri destinatari. Ad essi si impone il dovere di apprendere da fonti ufficiali. Nei casi qui in esame l'atto di comando viene presentato come un'ulteriore prescrizione di conoscenza obbligatoria. Si può in ogni modo osservare che altro è fissare una verità legale, ragion per cui spetta ai destinatari prendere effettiva conoscenza. Altro, invece, una comunicazione intesa a conseguire una conoscenza di fatto tra $\mathrm{i}$ consociati destinatari.

Più recente, oltre l'espressione di ordini e divieti, è lo sviluppo di attività di comunicazione pubblica volte a stabilire una maggiore interazione con i consociati, oltre la relazione comando-obbedienza. È ormai diffusa l'espressione "comunicazione del rischio" (risk communication) intesa a indicare quelle attività che contribuiscono a controllare e mitigare gli effetti del rischio, attraverso la condivisione di informazioni scientificamente corrette, tali da consentire ai consociati l'apprendimento di procedure, comportamenti di autoprotezione e buone pratiche da adottare durante l'eventuale insorgere del rischio ${ }^{17}$.

${ }^{17}$ Sul tema cfr. B. Reynolds, M.W. SeEger, Crisis and Emergency Risk Communication as an Integrative Model, cit., diffusamente; V.T. Covello, P. M. SANDMAn, Risk Communication: Evolution and Revolution, in A. Wolbarst (ed.), "Solutions to an 
Queste attività si svolgono principalmente nel tempo ordinario. Si tratta di una comunicazione ulteriore rispetto a quella immediata di risposta alla calamità, che si giustifica per la previsione del rischio e per la sua valutazione in una fase antecedente alla calamità stessa. Essa implica la stretta collaborazione delle autorità con la comunità scientifica, al fine di acquisire le indispensabili conoscenze della scienza e della tecnica, che rappresentano il presupposto per la predisposizione di misure (normative e provvedimentali) volte a garantire la correttezza, sotto il profilo scientifico, sia delle decisioni di politica pubblica sia dei comportamenti personali da approntare in condizioni emergenza.

Una tale comunicazione tende, inoltre, a promuovere attività di formazione e di educazione della collettività. Infatti, fine della comunicazione del rischio è conseguire la partecipazione attiva dei cittadini mediante la diffusione di informazioni utili a favorire cognizione dei rischi (risk awareness) e comportamenti responsabili, nella prospettiva di una società resiliente. Suggerito dall'ingegneria dei materiali, il termine resilienza è entrato nell'uso ad indicare la capacità di una comunità di adattarsi ai molteplici rischi.

D'altro canto, l'assunzione di atteggiamenti corretti da parte dei consociati può concorrere a rendere più efficace la risposta operativa, facilitando la gestione dell'emergenza. A questo proposito, nella comunicazione preventiva e precauzionale del rischio si può ravvisare perseguita altresì la finalità di pianificare la partecipazione e la collaborazione dei soggetti impegnati in attività di comunicazione durante la gestione dell'emergenza. In tal modo risultano fissati alcuni aspetti dell'organizzazione, a seconda della tipologia di rischio, sul piano di una sostanziale efficacia dell'azione amministrativa. Ciò nella prospettiva della costruzione di un'amministrazione resiliente.

Sia pure in termini empirici, in riferimento all'insieme delle attività

Environment in Peril", Baltimore, Johns Hopkins University Press, 2001, p. 64 sgg.. Secondo la definizione normativa, il rischio è la probabilita che un determinato evento si verifichi in un dato periodo o in circostanze specifiche (art. 3, lett. q), D.Lgs. n.105/2015). Per vero, vi sono difficoltà di pervenire ad una definizione comunemente accettata, a causa della complessità e "multidimensionalità" della stessa nozione, con conseguenze dirette sul dibattito in corso tra esperti, scienziati, decisori politici e consociati, come illustrato da A. Alemanno, Per una comprensione pluralistica del rischio. Prefazione a A. CERASE, "Rischio e Comunicazione. Teorie, modelli e problemi", Milano, Egea, 2017, p. 11. Per approfondimenti nella legislazione italiana si veda più oltre al paragrafo 3.2. 
di comunicazione (esterna e interna), compiute dalle autorità, in previsione o in presenza di eventi straordinari per i quali si pone la necessità di provvedere, anche in via preventiva e precauzionale, all'ordine, alla sicurezza, all'incolumità collettiva e a tutti gli eventuali interessi pubblici che possano venire meno, è possibile parlare di "comunicazione (pubblica) di emergenza", facendo però attenzione che detta nozione è mancante di una definizione di ordine legislativo.

Tuttavia, è dato di osservare che, anche in ragione di tale mancanza, non risulta un'univoca nozione di detta comunicazione, che dunque risente di una certa variabilità a seconda dell' ambito di studi in cui viene enunciata. In effetti, in analogia con la comunicazione pubblica, anche la comunicazione di emergenza ha una natura interdisciplinare, in raccordo e confronto con discipline diverse ${ }^{18}$. Riesce però ancora incerto combinare le applicazioni di differenti ordini di pensiero e di pratica in questo nuovo settore di ricerca e di didattica.

In ogni modo, vi è di comune il contesto straordinario di riferimento: il "manifestarsi di una situazione pericolosa che richiede attività e provvedimenti specifici, urgenti, necessari ed eccezionali", tra i quali il bisogno di informare e allertare la popolazione e le amministrazioni preposte a tali fini ${ }^{19}$. Tale nozione di emergenza è stata fissata nella regolazione per la gestione di crisi di sicurezza nazionale (di competenza della difesa civile), come risulta dall'elaborazione di un quadro semantico comune a livello interministeriale. L'uso di un'espressione tanto ampia e generica risponde alla difficoltà di prevedere in singoli termini specificati tutte le possibili singole situazioni di emergenza.

\section{Disciplina nel diritto positivo}

Le regole rilevanti per la comunicazione di emergenza risultano in poche disposizioni, prodotte da fonti diverse: la legge $n .150$ del 7 giugno 2000, "Disciplina delle attività di informazione e di comunicazione delle pubbliche amministrazioni”, e altre leggi con oggetti in materie eterogenee.

18 M. Di Benedetto, Comunicazione (IV Comunicazione pubblica) in Enc. giur., X, aggiornamento, Roma, 2002, p. 1.

${ }^{19}$ Art. 2, lett. b), D.P.C.M., 5 maggio 2010, “Organizzazione nazionale per la gestione di crisi”. 
a) L'anzidetta legge n. 150 è tutt'oggi il primo e unico atto normativo che attiene al complesso delle attività di comunicazione pubblica, in ragione dei principi del più generale processo di riforma dell'amministrazione. Tale legge è di contenuto sostanzialmente organizzativo, con la sola disciplina di attività e strutture e ampi rinvii al potere regolamentare del Governo e delle amministrazioni, senza indicazioni rigorose sui contenuti e sui tempi di attuazione ${ }^{20}$. È da notare che nella suddetta legge mancano disposizioni specifiche per i casi di emergenza.

b) Sono anche da menzionare le norme di disciplina degli organi costituzionali, delle pubbliche amministrazioni dello Stato e di altri enti territoriali, integrate dai regolamenti interni. Per tali disposizioni risulta delineata la cd. "comunicazione istituzionale", comprensiva di quelle doverose e complesse attività riguardanti la circolazione delle informazioni e dei dati giuridicamente rilevanti per le singole istituzioni, nei loro rapporti reciproci e nei rapporti con i cittadini ${ }^{21}$. Si aggiunga che nella suddetta nozione rientrano anche le attività di esternazione connesse all'indirizzo politico della maggioranza. In proposito va ricordato che durante le situazioni di emergenza, conformemente ai principi costituzionali e all'esigenza del diritto all'informazione, il Presidente del Consiglio e i Ministri portano a conoscenza dei consociati le decisioni assunte e i servizi predisposti, sotto la responsabilità politica del Governo, per la tutela dei più rilevanti diritti e interessi, salute, sicurezza e ordine pubblico $^{22}$. A tale fine operano congiuntamente: il Segretariato generale, l'Ufficio stampa e del Portavoce del Capo del Governo presso la Presidenza del Consiglio, i servizi del Sistema di informazione per la sicurezza della Repubblica e gli uffici preposti dei Ministeri.

20 Per approfondimenti sia consentito rinviare a S. ILLARI, Informazione e comunicazione pubblica: strutture e strumenti dell'Amministrazione, in G. AvANZINI, G. MatUCCI, L. Musselli, "Informazioni e media nell'era digitale”, Milano, Giuffrè, 2019, p. 277.

${ }^{21}$ P. Marsocci, Poteri e pubblicità. Per una teoria giuridica della comunicazione istituzionale, cit., p. 163. Nella teoria si è soliti ripartire la comunicazione pubblica negli ambiti più specifici della comunicazione istituzionale, politica e sociale.

22 La regolazione in norme di dette attività è rinvenibile in alcune disposizioni della L. 23 agosto 1988, n. 400 e del Regolamento interno del Consiglio dei Ministri (D.P.C.M. 10 novembre 1993). Da ultimo v. ricostruzione di E. Longo, M. MALvicini, Il decisionismo governativo: uso e abuso dei poteri normativi del Governo durante la crisi da COVID19 , in federalismi.it, n. 28, 2020, p. 224 sgg.. 
c) Infine, pur mancando un'apposita disciplina legislativa generale circa la comunicazione del rischio e dell'emergenza, disposizioni in materia sono rinvenibili in alcune normative di settore, non estese in via generale a tutto l'ordinamento interno. Fra l'altro, in virtù del diritto positivo dell'Unione europea, vi sono regole stabilite per particolari ordini di rapporti. Si ricorda, ad esempio, il rischio industriale, con apposita regolazione della comunicazione preventiva e in emergenza, il rischio da esposizione ai campi elettromagnetici, la sicurezza alimentare. Vi sono anche norme di rango primario, per vero rare, nelle quali sono previste attività di informazione collegate a funzioni pubbliche in determinati contesti emergenziali come la protezione civile.

\subsection{Di alcune questioni della legge n. 150 del 2000}

A vent' anni dall'entrata in vigore della legge sulla comunicazione pubblica pare da accennare ad alcuni aspetti problematici, che possono avere effetto proprio sulla comunicazione di emergenza.

a) In primis, l'assenza di controlli e di sanzioni per le istituzioni e le amministrazioni inadempienti rispetto alle regole circa la predisposizione di strutture specializzate in ogni amministrazione, a livello statale, regionale e locale, con personale qualificato professionalmente. Tali sono: l'ufficio relazioni con il pubblico per la gestione delle attività di comunicazione; l'ufficio stampa e il portavoce, per le competenze relative all'informazione verso i mass media ${ }^{23}$. Peraltro, al presente si tratta di un'organizzazione ormai superata, che risale ad un'epoca in cui nelle amministrazioni pubbliche non era in uso né il web né altre tecnologie digitali dell'informazione e della comunicazione. In ogni modo, la formazione di queste strutture è avvenuta con forte ritardo e in maniera eterogenea ${ }^{24}$. Spesso, anche per mancanza di risorse eco-

23 Per approfondimenti S. ILLARI, Informazione e comunicazione pubblica: strutture e strumenti dell'Amministrazione, cit., p. 285.

${ }^{24}$ Per vero, gli unici dati ufficiali risalgono al monitoraggio del primo quinquennio, che evidenziava l'attuazione della legge per circa metà delle Pubbliche amministrazioni V. Rapporto al Ministro per la funzione pubblica sulla situazione e le tendenze della comunicazione istituzionale in Italia, realizzato dall'Università IULM di Milano, coordinamento di 
nomiche, è stata rilevata la carenza di personale dotato delle richieste competenze professionali. Ne è derivata un'attuazione parziale e disordinata della legge, detta "a macchia di leopardo".

b) Si aggiunga altresì che tali strutture specializzate risultano non solo distinte ma, di fatto separate, nonostante si fosse tentato con la cd. "direttiva Frattini" di favorire forme di interazione e di sinergia 25. Più in generale, si rileva la mancata previsione di forme di coordinamento, anche tra le amministrazioni. Nessun criterio sul punto è dato desumere dalla legge. Spesso le amministrazioni pubbliche si muovono ad libitum, senza linee-guida o protocolli di coordinamento, al verificarsi di eventi straordinari. Pare che ne abbiano a derivare conseguenze gravi sull'efficacia della risposta operativa. In effetti, in condizioni di emergenza, anche per l'amministrazione più tempestiva ed efficiente nel comunicare potrebbero manifestarsi difficoltà di comunicazione eventuali, ma di fatto possibili, di altre amministrazioni. Potrebbe anche conseguire danno per l'effettiva protezione dei consociati, con possibili questioni di responsabilità degli amministratori pubblici.

c) Infine, è da evidenziare il mancato adeguamento della disciplina normativa alle trasformazioni dovute all'uso delle tecnologie digitali, che pure sono previste dalla legge con generico riferimento all"utilizzo di "strumenti telematici" ma senza specifico obbligo o forme d'incentivazione. In ogni modo, risulta di tutta evidenza che in situazioni di emergenza sia imposta la necessità di applicare tali mezzi di comunicazione immediata e interattiva nell' interesse generale. Tuttavia, è da notare che al presente vi è solo un esplicito invito all'uso di detti strumenti da parte delle pubbliche amministrazioni. Tale è il punto 8.2. della circolare n. 2 del 30 maggio 2017 sulla trasparenza ("Attuazione delle norme sull'accesso civico generalizzato $\mathrm{cd}$. FOIA"), dovuta al Ministro per la semplificazione e la pubblica amministrazione: "le pubbliche amministrazioni sono invitate a valorizzare il dialogo con le comunità di utenti dei social media (Facebook, Twitter, ecc.)".

S. Rolando, Milano, FrancoAngeli, 2005. Ma è l'esperienza, specie dei piccoli Comuni, a confermare l'inattuazione.

${ }^{25}$ Spec. artt. 2 e 3 dir. "Attività di comunicazione delle pubbliche amministrazioni”, emanata dal Ministro per la funzione pubblica il 7 febbraio 2002. 
Così pare importante la revisione della legge, come già riconoscibile dall'opera compiuta dal Dipartimento della Funzione Pubblica per "l'amministrazione aperta": nel gennaio 2020 si è costituito un gruppo di lavoro con il compito di elaborare linee guida per una social media policy nazionale e proposte per avviare un percorso di riforma della legge n. 150 del 2000. Un primo documento d'intenti è già stato presentato nel giugno 202026 .

A parte l'eventuale adeguamento di linguaggio e di strumentazione digitali, è importante notare che non risultano intervenute ulteriori disposizioni di legge dirette al perfezionamento della disciplina fissata nel 2000. Per condivisa opinione, non pare da lasciare ai margini la comunicazione pubblica, quasi attività residuale relegata al solo aspetto organizzativo. Vale ricordare che parte della dottrina ha ritenuto che la stessa sia da intendere come una funzione amministrativa in senso proprio, determinante per il perseguimento del fine dell'amministrazione ${ }^{27}$. Con ciò si intende non solo un'attività specialistica, di particolari settori, ma anche funzione di carattere diffuso, esercitata dall'amministrazione pubblica nel suo complesso 28 .

\subsection{Applicazioni della comunicazione di emergenza}

Come già accennato, nell'ordinamento italiano la comunicazione del rischio e dell'emergenza trova sistematica disciplina in pochi settori. Si tratta di previsioni generali, che implicano attuazione mediante provvedimenti amministrativi specifici di singoli organi competenti.

${ }^{26}$ Il documento e la relazione di accompagnamento sono consultabili in rete sul sito web del Ministero per la funzione pubblica.

27 G. ARENA, La funzione pubblica di comunicazione, in G. ARENA (a cura), "La funzione di comunicazione nelle pubbliche amministrazioni”, Rimini, Maggioli, 2001, p. 29; G. AzZARITI, La comunicazione come funzione, Introduzione a G. ARENA (a cura), "La funzione di comunicazione nelle pubbliche amministrazioni”, Rimini, Maggioli, 2001, p. 15; F. MERLONI, Sull'emergere della funzione informativa delle pubbliche amministrazioni, in F. Merloni (a cura), "L'informazione delle pubbliche amministrazioni”, Rimini, Maggioli, 2002, p. 15.; B. MATTARELla, Informazione e comunicazione amministrativa, in Riv. trim. dir. pubbl., n. 1, 2005, p. 1.

${ }^{28}$ G. ArenA, La funzione pubblica di comunicazione, cit., p. 80 sgg.. 
a) In forza del diritto dell'Unione europea, è stabilito per il rischio industriale un quadro normativo in continuo aggiornamento. Nelle cd. "direttive Seveso", sul controllo dei pericoli causati da incidenti rilevanti per la fuoriuscita di determinate sostanze pericolose, è contemplata la predisposizione di un complesso di attivita da parte di vari soggetti pubblici e privati (le stesse industrie coinvolte), chiamati a collaborare e a coordinarsi, secondo dettagliate disposizioni, che ricomprendono anche la comunicazione del rischio e alcuni obblighi di informazione. Tali disposizioni sono state recepite nell'ordinamento italiano dal $1988^{29}$.

Si deve osservare che nella legislazione in esame il Comune dove è posto l'impianto esercita attribuzioni in materia d'informazione, consultazione e partecipazione dei consociati ai processi decisionali. In particolare, stante il riconoscimento del diritto di accesso alle informazioni, spetta all'autorità comunale mettere "tempestivamente" e "permanentemente" a disposizione del pubblico, con un linguaggio comprensibile, le informazioni fornite dal gestore dell'impianto, riportando, in formato elettronico e con pubblicazione sul sito web del Comune, almeno quelle relative allo stabilimento, alle sostanze impiegate nel processo di produzione e agli scenari di rischio ${ }^{30}$.

Quanto alla diffusione delle suddette informazioni al pubblico, tale compito è attribuito al Sindaco che fornisce direttamente alla popolazione, nella forma più idonea, anche indicazioni chiare e comprensibili circa le misure di sicurezza e la condotta da tenere in caso di incidente. Allo stesso modo, è prescritta la diffusione delle informazioni in strutture e aree pubbliche, che potrebbero essere

${ }^{29}$ Dir. 82/501/CEE, la cd. "Seveso I", recepita in Italia con il D.P.R. 17 maggio 1988, n.175, "Attuazione della direttiva CEE n. 82/501, relativa ai rischi di incidenti rilevanti connessi con determinate attività industriali, ai sensi della legge 16 aprile 1987, n. 183". Successive modifiche sono state introdotte, con l'obiettivo di migliorare il quadro normativo sulla base delle esperienze acquisite. Si ricordano, in particolare, la dir. 96/82/CE, definita "Seveso II" e recepita con D.Lgs. 17 agosto 1999, n. 334; la dir. 2003/105/CE, recepita con D. Lgs. 21 settembre 2005, n. 238; in ultimo la dir. 2012/18/UE, definita "Seveso III", recepita con D. Lgs. 26 giugno 2015, n. 105. Con l'adozione di quest'ultimo decreto si è provveduto a completare la frammentata normativa preesistente, quasi redigendo un vero e proprio "testo unico" in materia di incidenti rilevanti.

30 Art. 23, c. 6, D. Lgs. n. 105/2015. 
interessate per possibili incidenti (scuole, ospedali e stabilimenti adiacenti) ${ }^{31}$.

Si aggiunga che le informazioni da trasmettere sono elaborate sulla base di linee guida fornite dal Dipartimento della protezione civile $^{32}$. Per tali informazioni è altresì stabilito un aggiornamento almeno ogni cinque anni.

Inoltre, allo scopo di limitare le conseguenze provocate da incidenti rilevanti, sono da predisporre appositi piani di emergenza per ogni stabilimento industriale: il piano di emergenza interna, di competenza del gestore, nel quale si individuano le azioni che i lavoratori e le squadre di soccorso devono compiere in caso di incidente e le modalità d'informazione per gli stessi lavoratori; il piano di emergenza esterno, nel quale si delineano e si coordinano azioni dei soggetti coinvolti nella gestione degli incidenti rilevanti, allo scopo di ridurre i danni e informare la popolazione dell'evento in corso, nelle modalità concordate anche con il Sindaco del Comune dove ha sede lo stabilimento ${ }^{33}$. Il piano di emergenza esterno è di competenza esclusiva del Prefetto, che lo redige seguendo apposite linee guida del Dipartimento della protezione civile, d'intesa con le Regioni e gli enti locali interessati, sentito il Comitato tecnico regionale e previa consultazione della popolazione. Ė ancora il Prefetto a coordinarne altresì l'attuazione. È da notare che nel piano di emergenza esterno è inserita anche una specifica sezione denominata "informazione alla popolazione", posta al fine di pianificare il quadro delle attività per la gestione dell'informazione, sulla base di specifiche linee guida redatte dal Dipartimento protezione civile, in raccordo con i piani di emergenza interna e sulla scorta delle informazioni trasmesse dal gestore degli impianti ${ }^{34}$. È previsto l'aggiornamento del piano ogni tre anni.

Infine, sono stabiliti protocolli e flussi informativi da seguire in caso di incidente rilevante: è il gestore dello stabilimento che, uti-

${ }^{31}$ Art. 23, c. 7, D. Lgs. n. 105/2015.

${ }^{32}$ Le linee guida sull'informazione alla popolazione attualmente in vigore sono state stabilite nel novembre 2006 con D.P.C.M. 16 febbraio 2007.

33 Art. 21, D. Lgs. n. 105/2015.

${ }^{34}$ Le linee guida attualmente in vigore sono state stabilite nel dicembre 2004 con il D.P.C.M. 25 febbraio 2005. 
lizzando i mezzi più adeguati, è tenuto ad informare una serie di autorità pubbliche, tra le quali in primis il Prefetto. Quest'ultimo, nel disporre l'attuazione del piano di emergenza esterna e nel garantire che siano adottate le misure di emergenza necessarie, informa, tramite il Sindaco, le persone potenzialmente soggette alle conseguenze dell'incidente rilevante, anche con riguardo alle eventuali misure intraprese per attenuarne le conseguenze. Nel contempo, lo stesso informa immediatamente i Ministeri e taluni uffici pubblici interessati, la Regione, nonché i Prefetti competenti per gli ambiti territoriali limitrofi 35 .

b) Anche in riferimento alla funzione di protezione civile - volta a tutelare la vita, l'integrità fisica, i beni, gli insediamenti, gli animali e l'ambiente dai danni o dal pericolo di danni derivanti da calamità naturali o di origine antropica - da tempo sono in vigore elementi di una disciplina legislativa sulla comunicazione in emergenza. Per vero, a questa funzione da sempre sono correlati obblighi di informazione a carico delle autorità per i soccorsi alle popolazioni colpite da eventi calamitosi e per la gestione dell'emergenza con le amministrazioni interessate, nella fase immediatamente successiva all'evento ${ }^{36}$. Tuttavia, è da notare che tale impostazione tradizionale è mutata con l'approvazione della legge n. 225 del 1992, istitutiva del moderno Servizio nazionale della protezione civile, attraverso la quale il legislatore ha ampliato le attività in capo al suddetto Servizio, stabilendo anche il compito di prevedere e prevenire il verificarsi di possibili fenomeni calamitosi. Si è così introdotto un nuovo modello finalizzato alla protezione della popolazione prima del verificarsi dei fenomeni, che si fonda sulla comunicazione pre-

35 Art. 25, D. Lgs. n. 105/2015.

${ }^{36}$ Ad es. nel R.D.L. del 9 dicembre 1926, n. 2389, "Disposizioni per i servizi di pronto soccorso in caso di disastri tellurici o di altra natura" erano previste attività di comunicazione del Ministro degli Interni, che di tale gestione assumeva la gestione e il coordinamento, con il Presidente del Consiglio e i Ministri. Ciò allo scopo di effettuare una prima ricognizione sui luoghi del disastro e di predisporre l'organizzazione necessaria, con l'ordine alle autorità civili e militari e al personale dei ministeri e della Croce Rossa, che giungevano sul posto, di mettersi a disposizione dello stesso Ministro. Nel contempo, ordini di vario tipo venivano impartiti per la tutela della pubblica incolumità, in primis da parte del Prefetto, che provvedeva alla direzione e al coordinamento dei servizi fino all'arrivo sul luogo del disastro del Ministro degli Interni, ma anche da parte del funzionario del Genio civile più elevato in grado e da parte delle autorità civili e militari impegnate nei soccorsi. 
ventiva del rischio, la formazione e la partecipazione dei consociati $^{37}$. Si tratta di un modello poi perfezionato con l'entrata in vigore del Codice di protezione civile del 2018, che, pur mantenendo l'assetto delineato dalle norme della precedente legge n. 225 del 1992 e molteplici successive modifiche, ha rinnovato nel suo complesso questa materia di competenza legislativa concorrente, con una disciplina completa e organica che si propone di fissare il quadro generale dei principi fondamentali e degli indirizzi ${ }^{38}$.

Benché l'informazione alla popolazione, così come la comunicazione di protezione civile intesa in senso lato, sia un'attività che si declina su diverse fasi del ciclo di emergenza (in ordinario, nella fase di allertamento, a emergenza in atto), in continuità con la precedente disciplina, nell'attuale quadro normativo tale attività non ha una collocazione propria nel nuovo Codice ma è solo nominata in riferimento alla gestione dei soccorsi durante l'emergenza in atto e poi ancora tra le misure di prevenzione da attuare "in tempo di pace", quando non c'è un'emergenza attiva e si lavora alla pianificazione degli interventi e al modello comunicativo ${ }^{39}$. In particolare, nell' ambito delle attività di prevenzione, finalizzate ad evitare o ridurre al minimo la possibilità che si verifichino danni, conseguenti agli eventi calamitosi, anche sulla base delle conoscenze acquisite per effetto delle attività di previsione, permane l'articolazione tra misure "strutturali" e "non strutturali", che pare più ampia, esplicita e chiara ${ }^{40}$. Nel secondo gruppo è inclusa "l'informazione alla popolazione sugli scenari di rischio e le relative norme di comportamento nonché sulla pianificazione di protezione civile" 41 . Si tratta di un'indicazione più puntuale

${ }^{37}$ L. 24 febbraio 1992 n. 225, "Istituzione del servizio nazionale di protezione civile".

${ }^{38}$ D.Lgs. 2 gennaio 2018, n. 1, "Codice di protezione civile", in attuazione dei principi contenuti nella legge di delega per il riordino, non meramente compilativo, della disciplina in materia (L. 16 marzo 2017, n. 30). Pubblicato sulla Gazzetta ufficiale il 22 gennaio 2018, il Codice è entrato in vigore il 6 febbraio dello stesso anno. Successivamente, con il D. Lgs. 6 febbraio 2020, n. 4, sono state emanate disposizioni integrative e correttive, specie in rapporto ad aspetti di governance e al coordinamento fra la fase emergenziale e quella di ritorno all'ordinarietà. Per approfondimenti si rimanda a U. AlLEgretTI, Il Codice della Protezione Civile, in Aedon, n. 1, 2018; V. PEPE, La gestione dei rischi nel codice della protezione civile, in federalismi.it, n. 1, 2020, p. 213.

39 Art. 2, c. 4 , lett. f) e c. 6.

40 Art. 2 , c. 4 e 5.

${ }^{41} \mathrm{Sul}$ punto è da notare che, tra gli indirizzi specificati dai principi di delega per la ri- 
che, per vero, si completa e va letta in correlazione ad altri elementi:

- la novità data dall'inserimento nel Codice della cd. "tipologia dei rischi di protezione civile" e dalla previsione di ulteriori rischi per i quali è possibile esercitare l'azione del Servizio nazionale, pur nel rispetto delle competenze attribuite nell'ordinario ai soggetti indicati nelle normative di settore ${ }^{42}$.

- l'indicazione di altre significative "attività di prevenzione non strutturale" ricomprese nel lungo elenco: "l'allertamento del Servizio nazionale", "la pianificazione di protezione civile", "la diffusione della conoscenza e della cultura di protezione civile, anche con il coinvolgimento delle istituzioni scolastiche, allo scopo di promuovere la resilienza delle comunità e l'adozione di comportamenti consapevoli e misure di autoprotezione da parte dei cittadini" ${ }^{43}$. Si tende quindi a sviluppare una "cultura" di protezione civile attraverso l'intero complesso di misure da mettere in atto per ridurre l'esposizione al rischio.

- la disciplina tecnico-operativa per tutte le attività di "prevenzione non strutturale" del rischio che - con esplicito riguardo sia alla comunicazione tra componenti e strutture operative del Servizio nazionale sia all'informazione alla popolazione - risulta demandata alla pianificazione dei vari livelli territoriali, in raccordo con gli strumenti di pianificazione territoriale, secondo apposita direttiva del Presidente del Consiglio dei Ministri, che, in qualità di autorità nazionale e titolare delle politiche di protezione civile, assicura l'indirizzo unitario ${ }^{44}$. In effetti, l'intervento statale è giustificato al fine di garantire il necessario coordinamento e l'integrazione tra i diversi territori, pur nel rispetto dell'autonomia organizzativa delle Regioni e delle Province autonome, cui spetta, in ogni modo, di assicurare la predisposizione e l'attuazione delle attività volte alla previsione e prevenzione dei rischi ${ }^{45}$.

forma in materia, il Parlamento aveva indicato la necessità di garantire un quadro coordinato e chiaro su tutto il territorio nazionale in tema di comunicazione del rischio (art. 1, c. 2, lett. d), L. n. 30/2017).

42 Art. 16.

43 Ancora in riferimento ai principi della legge delega al Governo, era stata esplicitamente fissata la necessità di promuovere la resilienza dei consociati attraverso molteplici attività di "prevenzione non strutturale" (art. 1, c. 1 lett. d), L. n. 30/2017).

${ }^{44}$ Art. 18, dedicato interamente alla pianificazione di protezione civile.

45 Art. 11, c. 1 . In riferimento alla prevenzione dei rischi e alla pianificazione di 
Infine, è dato di notare un aspetto essenziale che caratterizza la nuova normativa di protezione civile, sulla scorta del modello di "amministrazione condivisa" secondo cui i destinatari delle comunicazioni devono oggi considerarsi non più come semplici amministrati in chiave autoritaria, ma soggetti attivi da coinvolgere e con i quali rapportarsi in modo più diretto ${ }^{46}$. Si tratta della partecipazione attiva dei cittadini, singoli o associati, alle attività di protezione civile quale passaggio essenziale per l'accrescimento della resilienza delle comunità, nella misura in cui anche tale partecipazione possa contribuire a fornire informazioni utili per una maggiore risk awareness e l'adozione di comportamenti consapevoli di autoprotezione ${ }^{47}$. Questa catena di partecipazione e diffusione delle informazioni - consapevolezza - autoprotezione - maggiore resilienza della comunità costituisce una delle più significative novità introdotte dal Codice, fissando normativamente che gli obblighi informativi a carico della pubblica amministrazione hanno una finalità specifica che è quella di mettere il cittadino in condizione di comportarsi in modo da contribuire direttamente e personalmente alla riduzione della propria esposizione ai rischi.

In particolare, si aggiunga che, fermi restando tutti gli obblighi di informazione in capo all'amministrazione pubblica e segnatamente delle autorità di protezione civile, nel Codice è stato statuito il dirittodovere di autoprotezione, dapprima promosso in sede giurisprudenziale: "le componenti del Servizio nazionale, nell'ambito delle rispettive attribuzioni, forniscono ai cittadini informazioni sugli scenari di rischio e sull' organizzazione dei servizi di protezione civile del pro-

protezione civile del territorio, è dato di notare che il Consiglio di Stato, nel formulare il parere allo schema di decreto, aveva insistito sulla necessità non solo di prevedere le relative azioni, ma anche di monitorare attentamente le attività effettivamente compiute. In particolare, rilevando che "le best practices internazionali della protezione civile, e la stessa ratio della delega, considerano l'attività di previsione, prevenzione, pianificazione di pari, se non maggiore, importanza rispetto a quella di gestione delle emergenze", nel parere era suggerito l'inserimento nel testo di una norma apposita per verificare, ex post, i risultati dell'attività di prevenzione. In Consiglio di Stato, Sez. cons. atti norm., 19 dicembre 2017, n. 2647, 11. Il testo del parere è reperibile al sito internet https://www.giustizia-amministrativa.it.

46 Sul punto G. ARENA, Comunicazione e amministrazione condivisa, in S. RolANDo (a cura), "Teorie e tecniche della comunicazione pubblica. Dallo Stato sovraordinato alla sussidiarietà", Milano, Etas, 2002, p. 45.

${ }^{47}$ Art. 2, c. 1 , lett. e) ed f), e art. 31, c. 1 e 2. 
prio territorio, anche al fine di consentire loro di adottare misure di autoprotezione nelle situazioni di emergenza (...), in occasione delle quali essi hanno il dovere di ottemperare alle disposizioni impartite dalle autorità di protezione civile in coerenza con quanto previsto negli strumenti di pianificazione"48. Vi è quindi motivo di considerare il ruolo delle attività di informazione (e pure di formazione) preventiva, adottate nei confronti di tutti i cittadini quale presupposto delle successive informazioni da diramarsi al verificarsi dell'evento, quasi "anelli" della medesima catena di protezione e di comunicazione.

\section{Cenni all'emergenza epidemiologica da Covid-19}

In sede di intervista radiofonica il professore Fulco Lanchester dell'Università "La Sapienza" di Roma ha rilevato che in Italia l'emergenza sanitaria, statuita con dichiarazione di apposito stato il 31 gennaio 2020, si sovrappone a quella politico-costituzionale, che, invece, è di più lunga durata, con un momento di particolare rilevanza in rapporto alle elezioni politiche del 2018. Allora, dopo una lunga crisi istituzionale, si è avuta la formazione di una maggioranza fondata sul cd. "contratto per il cambiamento" tra Movimento Cinque Stelle e Lega Nord. È seguita la formazione di una nuova maggioranza, con il Movimento Cinque Stelle e altri diversi partiti, esigua al Senato49. È pure da accennare a successive vicende, sul finire del gennaio 2021, onde è derivata la crisi anche di questa seconda maggioranza di governo.

Effetti problematici possono svilupparsi in questa inconsueta situazione di "doppia emergenza", riscontrabili anche nella comunicazione pubblica. Soprattutto nei primi mesi della pandemia si è registrata una frettolosa crescita di comunicazioni istituzionali da parte di autorità pubbliche e di esperti: il Presidente del Consiglio, Ministri, il Capo del Dipartimento della Protezione civile, Presidenti delle Regioni, i Sindaci,

48 Art. 31, c. 2. Il diritto-dovere di protezione civile è richiamato in più pronunce. V. p. es. Cass. Civ. sez. VI, 30/10/2018, n.27724, inedita. Si aggiunga che è stato il Parlamento che, in sede di legge delega di riordino della normativa in materia di protezione civile, ha introdotto una sottolineatura del tema dei doveri dei cittadini accanto ai loro diritti in termini di sicurezza (art. 1, c 1, lett. d), L. n. 30/2017).

49 Intervista a Fulco Lanchester di Roberta Jannuzzi, 26 ottobre 2020, reperibile sul sito web di Radio Radicale. 
il Consigliere del Ministro della salute per l'emergenza Covid-19, membri del Comitato tecnico-scientifico con funzioni di consulenza al Capo del Dipartimento della Protezione civile e al Ministro per la Salute, il Presidente e ricercatori dell' Istituto superiore di sanità. Fra l'altro, il Presidente della Repubblica, il 5 marzo 2020, con poche e severe parole, aveva raccomandato di non destare paure, di non cedere ad affermazioni poco scientifiche; altresì aveva ammonito di uniformarsi a spirito di concordia e unità di intenti nell'impegno per sconfiggere il virus.

La mancanza di coordinamento nei messaggi parrebbe indice di una gestione della comunicazione per certi versi improvvisata e non concordata, anche perché, come già accennato, non risultano prestabilite regole appropriate per la comunicazione pubblica quanto alle relazioni tra le autorità.

Particolari difficoltà appaiono in relazione al fatto che furono rese note le bozze dei provvedimenti, prima che questi ultimi fossero attuati. Si ricorda che nella serata del 7 marzo 2020 iniziò a circolare sui mezzi di informazione la bozza del decreto del Presidente del Consiglio dei Ministri (D.P.C.M.), che "chiudeva" la Lombardia e altre 14 province. Ne era seguita confusione e incertezza tra i cittadini. Si ebbe così notizia che in poche ore numerose persone erano affluite soprattutto nelle stazioni ferroviarie di Milano, dirette ai treni verso il Sud, prima dell'entrata in vigore delle nuove norme, causando assembramenti nocivi per la propagazione del virus. In questo contesto ansiogeno, a notte fonda, il Presidente del Consiglio fu costretto a convocare una conferenza stampa per spiegare i dettagli del nuovo decreto ${ }^{50}$. L'esempio corrisponde a quanto indicato dall'oms per cui la comunicazione è strettamente connessa alla tutela della salute pubblica. Ma è ancora lo stesso esempio ad evidenziare che la forza della comunicazione di emergenza si situa nella tempestività e nella chiarezza delle sue forme. Diversamente si tratta di una comunicazione che genera ulteriore emergenza anziché comunicarla.

Durante l'emergenza l'annuncio dei provvedimenti in apposite conferenze stampa del Presidente del Consiglio dei Ministri è diventato abituale. In proposito, è ancora il Professore della Sapienza a definire

${ }^{50}$ V. Dichiarazioni alla stampa del Presidente del Consiglio, Giuseppe Conte, sulle nuove misure del Governo per il contrasto e la prevenzione della diffusione del virus Covid19, 8 marzo 2020, reperibile alla voce "Galleria foto e video". 
i D.P.C.M. come "strumento di comunicazione" del Governo indirizzato ai mass media: le conferenze stampa precedono i provvedimenti, cui possono seguire eventuali ulteriori modifiche in corso di formazione. Fra gli studiosi si è anche fatto cenno di un "ruolo mediatico" del Capo del Governo per mezzo delle suddette conferenze stampa ${ }^{51}$. Anche il Capo del Dipartimento della Protezione civile risulta aver fatto ampio uso di conferenze stampa. Come è noto, si è trattato di una comunicazione puntuale e costante, a cadenza quotidiana, con l'aggiornamento dei dati rilevanti sull'andamento dell'epidemia nelle Regioni interessate dal virus. Tale attività di comunicazione esterna rientra nel coordinamento degli interventi necessari a fronteggiare l'emergenza sul territorio nazionale. Si tratta di apposito coordinamento esercitato dal Capo del Dipartimento della protezione civile a seguito della dichiarazione dello stato di emergenza ${ }^{52}$. In questo contesto, il 4 marzo 2020 lo stesso Capo della protezione civile ha diramato le linee guida operative per la gestione dell'emergenza Covid-19, fissando altresì la "catena di comando e di controllo" relativa al "flusso delle comunicazioni e delle procedure da attivare", in relazione a quattro livelli di coordinamento territoriale: nazionale, regionale, provinciale e comunale. Forme di comunicazione istituzionale sono previste per il livello regionale, pure con l'attivazione di numeri verdi, e per il livello comunale attraverso il Centro operativo comunale dei Comuni interessati dall'epidemia, chiamati a rafforzare l'informazione alla popolazione "tramite i canali ordinariamente utilizzati" ${ }_{53}$.

$\mathrm{Al}$ termine delle considerazioni che precedono e oltre le ultime vicende sarebbe da procedere a confronti puntuali con esperienze di altri

${ }^{51}$ Oltre all'impiego del D.P.C.M., parrebbe indicato che dalla gestione dell'emergenza epidemiologica da Covid-19 debba risultare una "valorizzazione" della figura dello stesso Presidente, secondo tendenze già rilevate da E. LongO, M. MALVICINI, Il decisionismo governativo: uso e abuso dei poteri normativi del Governo durante la crisi da Covid-19, cit., p. 223.

${ }^{52}$ Con particolare riguardo al soccorso e all'assistenza della popolazione eventualmente interessata dal contagio, al potenziamento della funzionalità dei servizi pubblici necessari al superamento dell'emergenza, alla continuità di erogazione dell'assistenza sanitaria, in continuità con le misure urgenti già adottate dal Ministero della salute, al rientro in Italia dei cittadini che si trovano nei Paesi a rischio e al rimpatrio dei cittadini stranieri nei Paesi di origine esposti al rischio. V. art. 1, OCDPC n. 630 del 3 febbraio 2020.

53 "Misure operative di protezione civile per la gestione dell'emergenza epidemiologica da Covid-19”, Dipartimento della Protezione civile, 4 marzo 2020. 
ordinamenti, anche al fine di riconoscere talune insufficienze delle nostre discipline. In limine, è dato di notare che l'ordinamento tedesco dispone di norme specificamente poste per regolare le emergenze connesse al diffondersi di fenomeni epidemici. Si tratta dell'Infektionsschutzgesetz, legge che, entrata in vigore il $1^{\circ}$ gennaio $2001 \mathrm{e}$ modificata durante la pandemia da Covid-19, ha costituito la base giuridica delle misure per la prevenzione e per il controllo della diffusione del Coronavirus, adottate dal Governo federale e dai governi dei Länder. Si osservi che in essa sono anche contenute norme di principio sulla comunicazione del rischio e delle emergenze ${ }^{54}$. Scopo della legge è di "prevenire le malattie trasmissibili nell'uomo, di individuare le infezioni in una fase precoce e prevenirne l'ulteriore diffusione", favorendo la responsabilità individuale di ciascuno (art. 1). In tale contesto l'attività di informazione e di comunicazione, oltrechè quella di educazione circa i pericoli connessi alle malattie trasmissibili e le misure di prevenzione, risulta qualificata come "funzione pubblica" (art. 3).

\begin{abstract}
Not only in order to better manage but also to prevent an emergency situation, that can affect entire communities of people, communication is an integral part of any emergency response to protect the fundamental rights, the right to health and life in the first place. It is probably an irreplaceable tool. However, an examination of public law dedicated to emergency communication seems to encounter difficulties, given the scarcity of rules and the still initial attention of the constitutional doctrine. In this context the essay deals with analyzing the information and
\end{abstract}

communication activities carried out by the Italian public authorities in case of emergency (natural disasters or man-made accidents), in order to protect health and life but also in order to prepare and to make the emergency administration work.

Particular attention is paid to the importance of preventive risk communication, through the sharing of scientifically correct information between public authorities and citizens to learn procedures, self-protection behaviors and good practices to be adopted during the onset of risk.

54 V. par. 2 e 3 Infektionsschutzgesetz del 20 luglio 2000. 